\title{
Evaluacion De Dos Instrumentos De Medicion En La Determinación Del Área Basal En Pinus Radiata
}

\author{
Norma Lara Vásconez (Ing. For.), \\ Janneth Morocho (Dr. Math), \\ Santiago Lara (Ing. Agron.), \\ Oscar Guadalupe (Ing. For),
} ESPOCH / Escuela Superior Politécnica de Chimborazo, Ecuador

doi: 10.19044/esj.2017.v13n24p10 URL:http://dx.doi.org/10.19044/esj.2017.v13n24p10

\begin{abstract}
This study compares the diameter of data obtained from utilizing two measuring instruments (the caliper and diameter tape). This instrument is the two most commonly used instruments for measuring tree diameters in forest inventory procedures. The measurement of diameter has a high implication in the estimation of the basal area and stand volume. It is therefore imperative to ensure accurate measurement of the diameter of trees in a stand. This work evaluated data taken with these two diameter measuring instruments. In order to evaluate the performance of the measuring instruments, parallel measurement of the diameter at breast height (DBH) of a sample of Pinus radiata trees in a plantation were measured. Also, the basal area of the stand was determined for both instruments. It was concluded through the analysis of variance (ANOVA) that there is no significant ( $p$ $=0.958$ ) difference between the averages of the calculated basal areas from the diameter measurements obtained by the diameter tape and the caliper. The estimated value from the diameter tape was 0,04190829, while the caliper was 0,04145599 . Thus, the use of the two measurement tools led to similar results in the calculation of the cross-sectional areas of pine trees measured at breast height.
\end{abstract}

Keywords: DBH, Basal Area, diameter, caliper, diameter measuring tape

\section{Resumen}

El presente estudio se realizó en una plantación de Pinus radiata ubicada en las instalaciones de la ESPOCH, Riobamba, Chimborazo, el mismo tiene como objetivo comparar datos de medición de diámetros obtenidos con dos instrumentos de medición, como son, cinta diametrica y forcípula; instrumentos comúnmente utilizados para la toma de diámetros en 
procedimientos de inventariación forestal. Siendo las mediciones de longitudes las más importantes, en esta área, la medición del diámetro tiene una alta implicación en los resultados de cálculos de área basal y volumen de rodales, por lo cual este trabajo consiste en evaluar datos tomados con estos instrumentos en la medición de diámetros, los mismos que serán empleados para el cálculo de área basal. Para evaluar el comportamiento de los métodos de determinación del DAP a nivel de árbol se definieron la variable área basal. El análisis estadístico se realizó utilizando el programa estadístico Rstudio, donde se aplicó un análisis ANOVA, para concluir que no existe diferencia significante $(\mathrm{p}=0.958)$ entre el promedio de las áreas calculadas a partir del diámetro medido ya sea por cinta diametrica o por forcípula, por tanto el empleo de las dos herramientas de medición conducen a similares resultados en el cálculo de las áreas de las secciones transversales con forcípula $0,04145599 \mathrm{~m} 2$ y con cinta diametrica $0,04190829 \mathrm{~m} 2$ de árboles de pino medidos a la altura del pecho.

Palabras-claves: Diámetro, área basal, forcípula, cinta diamétrica

\section{Introduction}

Algunos aspectos de mediciones de diámetros y de los instrumentos diseñados para este fin lo señala Prodan, 1997, quien menciona que sería Cotta el primero en utilizar la forcípula por primera vez en 1804 y probablemente fue el propio Cotta quien idealizó el instrumento, el mismo autor afirma que en $1860-$ M. R. Pressler diseña la barrena para la medición de crecimientos diametrales y que en 1891 - Surge la Regla de Christen para la medición de alturas. Durante este siglo comienza la aplicación de técnicas estadísticas al Inventario Forestal y surgen la mayoría de los instrumentos de medición. Por otro lado, el austriaco Walter Bitterlich, en 1948 -desarrolla la teoría del muestreo angular; posterior a lo cual, en 1950 aparece la forcípula finlandesa que permite la medición de diámetros no accesibles, cercano a este periodo en 1951 - W. Bitterlich inventa el Relascopio que permite medir los diámetros y alturas de los árboles y hacer fácilmente muestreos angulares. (Prodan, 1997), consecuentemente en 1970 se empiezan a desarrollar, también en Finlandia, las primeras forcípulas digitales.

El diámetro es una medida básica en cualquier árbol, este va a servir para mediciones de área basal, volumen, crecimiento, clasificación entre otras. Dentro del diámetro de los árboles el más conocido es el diámetro a la altura del pecho o DAP el cual nos ayuda para conocer el diámetro que posee el fuste del árbol en estudio; esta medición se la realiza a una altura de 1.30 m sobre el nivel del suelo (Ugalde, 1981). Cuando la sección transversal a la altura del pecho de un árbol no tiene forma circular o forma elíptica, siempre 
habrá un componente de error en la determinación del DAP al usarse las fórmulas de cálculo del círculo o de la elipse. Sin embargo, si la forcípula o la cinta diamétrica se usaran en forma apropiada, ambos instrumentos deberían proveer resultados comparables (Clark, 2000), y para aumentar la precisión de las medidas debido a la forma del fuste se pueden tomar dos medidas que sean perpendiculares entre sí de las cuales se va a sacar un promedio que corresponderá al diámetro verdadero (Husch, 1982). En caso de no disponer de una cinta diamétrica se puede emplear una cinta común y tomar el dato, y éste dato se lo dividirá para pi $(\pi)$ para obtener el valor del diámetro. La cinta diamétrica es una herramienta que nos ayuda a estimar el diámetro de un árbol, usualmente, la cinta viene graduada por uno de sus lados en metros y centímetros, el mismo que nos va a permitir medir el largo de la línea que une un punto a otro, y por el otro lado una escala que nos va a indicar la medida del diámetro de un árbol, dicha escala está construida tomando en cuenta la relación geométrica entre perímetro y diámetro de un circulo (Monroy, 2000).

\section{Metodología}

El presente trabajó de investigación se realizó en un rodal de bosque de pino localizado en la Escuela Superior Politécnica de Chimborazo, en la Facultad de Recursos Naturales (Provincia de Chimborazo) a una longitud $78^{\circ} 41^{\prime} 13.41 " \mathrm{O}$; latitud $1^{\circ} 39^{\prime} 5.60^{\prime \prime S}$ y a una altitud $2849 \mathrm{msnm}$. El rodal corresponde a una plantación de Pinus radiata D. Don, ubicado sobre un terreno semiplano con un área de $17433,59 \mathrm{~m}^{2}$.

Las mediciones se realizaron en un rodal de Pinus radiata, que tienen una edad aproximada de 45 años. Los instrumentos utilizados fueron una forcípula de metal graduada en centímetros y una cinta diamétrica. Para ello se ha considerado una parcela de 725 árboles de pino, localizada en la Escuela Superior Politécnica de Chimborazo, y se ha seleccionado al azar una muestra de 20 árboles; posteriormente de estos 20 se ha seleccionado al azar 10 árboles que se midieron con cinta diamétrica y a los restantes con forcípula. Los resultados son los siguientes:

Tabla 1. Selección de árboles al azar

\begin{tabular}{cccc}
\hline No. & $\begin{array}{c}\text { CINTA } \\
\text { DIAMETRICA }\end{array}$ & No. & FORCÍPULA \\
\hline 1 & 0,04500138 & 11 & 0,02405282 \\
\hline 2 & 0,04369448 & 12 & 0,02644612 \\
\hline 3 & 0,07973671 & 13 & 0,02216708 \\
\hline 4 & 0,01654702 & 14 & 0,0263022 \\
\hline 5 & 0,02424757 & 15 & 0,03220623 \\
\hline 6 & 0,03148411 & 16 & 0,06881345 \\
\hline 7 & 0,03540314 & 17 & 0,04618632 \\
\hline 8 & 0,03843791 & 18 & 0,04082814 \\
\hline
\end{tabular}




\begin{tabular}{cccc}
\hline 9 & 0,04608498 & 19 & 0,04637698 \\
\hline 10 & 0,05844559 & 20 & 0,08118052 \\
\hline
\end{tabular}

El objetivo del presente estudio es determinar la exactitud relativa entre la forcípula y cinta diametrica en pinos. El factor de interés de la presente investigación son las dos herramientas de medición siendo la variable respuesta el área de la sección transversal conocida como área basal medida en un rodal de Pinus radiata.

Para el presente estudio se proponen las siguientes hipótesis:

HIPÓTESIS NULA: No existe diferencia entre el promedio de las áreas calculadas a partir del diámetro medido con cinta diamétrica y forcípula.

$$
\mu_{C D}=\mu_{F S}
$$

HIPÓTESIS ALTERNA: Existe diferencia entre el promedio de las áreas calculadas a partir del diámetro medido con cinta diamétrica y forcípula.

$$
\mu_{C D} \neq \mu_{F S}
$$

Para evaluar el comportamiento de los métodos de determinación del DAP a nivel de árbol se definieron la variable área basal. El análisis estadístico se realizó utilizando el programa estadístico Rstudio, donde se aplicó un análisis ANOVA. continuación.

El modelo estadístico utilizado se expresa en la ecuación descrita a

$$
y_{i j}=\mu+\tau_{i}+\beta_{j}+e_{i j}
$$

Donde $i=1,2 ; j=1,2,3,4$

$y_{i j}=$ área de la sección transversal del árbol correspondiente al $j-$ ésimo bloque, calculada mediante el diámetro medido por la $i-$ ésima herramienta.

$\mu=$ media general

$\tau_{i}=$ efecto de la $i$ - ésima herramienta

$\beta_{j}=$ efecto del $j$ - ésimo bloque

$e_{i j}=$ error experimental

Tabla 2. Identificación de los tratamientos y las variables asociadas por árbol o por hectárea.

\begin{tabular}{ccccc}
\hline \multirow{2}{*}{ VARIABLES } & \multicolumn{4}{c}{ TRATAMIENTOS } \\
\cline { 2 - 5 } & CD & MAD & FS & PF \\
\hline DAP & TCD & TMAD & TFS & TPF \\
Área Basal & TCD & TMAD & TFS & TPF \\
\hline
\end{tabular}




\section{Resultados}

Según los resultados observados del análisis de varianza, no existe suficiente evidencia para rechazar la hipótesis nula. Este resultado posiblemente no sea el mismo al medir diámetros de especies con que presentan formas irregulares en el fuste, como es el caso de especies de hoja ancha que presentan variaciones de forma inherente a la genética de los árboles.

El modelo fue validado, es decir, se probaron los supuestos de normalidad y homocedasticidad por los métodos analítico y gráfico.

De acuerdo al valor $\mathrm{p}$ igual a 0.06225 arrojado por el test de normalidad Shapiro-Wilk, no se rechaza la hipótesis de normalidad de los datos, esto se confirma con el gráfico.

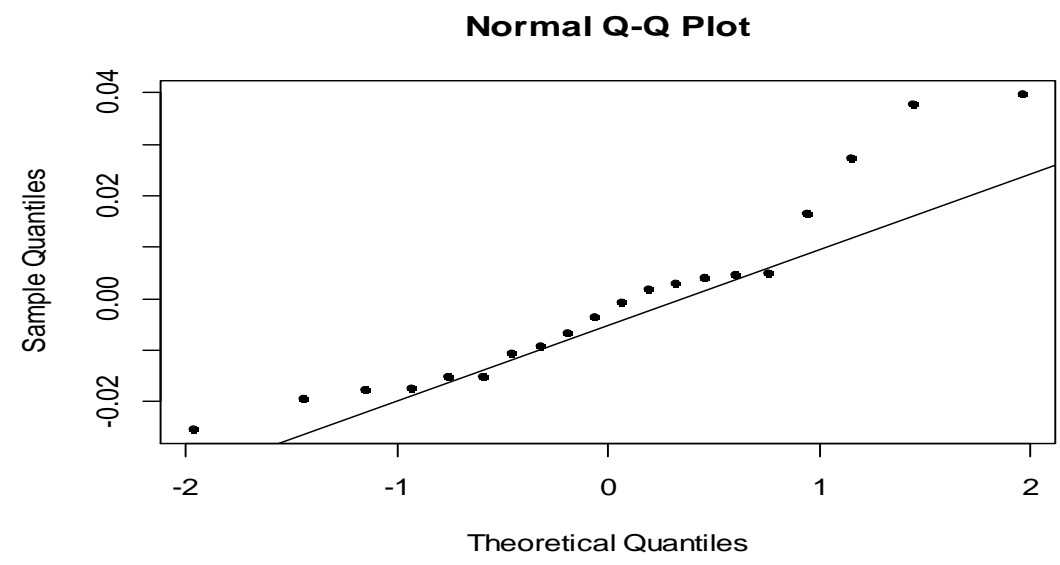

Fig. 1. Test de normalidad, según el test de Shapiro-Wilk, p-value $=0.06225$

La homogeneidad de las varianzas fue probada aplicando la prueba de Barlett, en la cual, no se rechaza la hipótesis de que las varianzas son iguales $p=0.7378$, gráficamente se observa de la siguiente manera. 


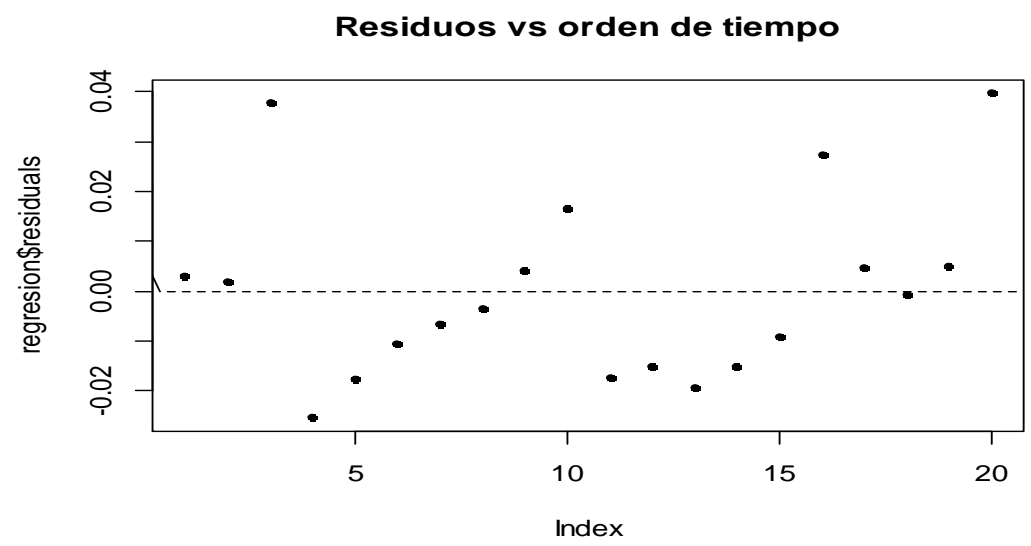

Fig. 2. Test de homogeneidad de varianzas, según Bartlett's p- value $=0.7378$

La hipótesis de independencia fue probada donde no se la rechaza según el test de Durbin Watson DW 2.617944, lo que se confirma con el gráfico, pues los puntos no siguen algún patrón, gráficamente la dependencia de los datos se muestra a continuación.

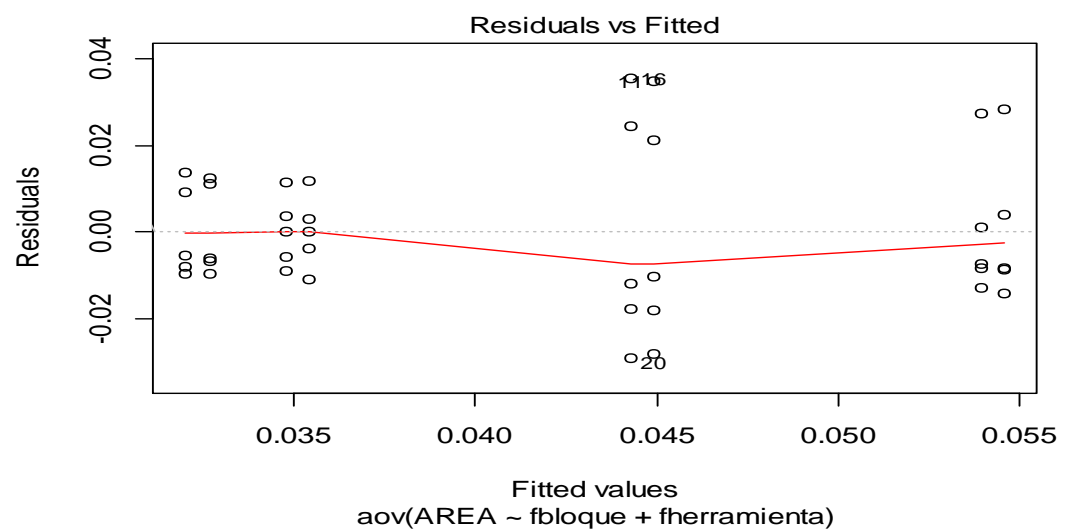

Fig. 3. Test de independencia según el test de Durbinwatson

\section{Conclusiones}

Mediante el análisis de datos se puede concluir que no puede ser rechazada la hipótesis nula ya que no existe diferencia entre el promedio de las áreas basales calculadas a partir del diámetro medido con cinta diametrica y el promedio de las áreas basales calculadas con forcípula; por tanto el empleo de las dos herramientas de medición conducen a similares resultados en el cálculo de las áreas de las secciones transversales de árboles de pino medidos a la altura del pecho. 
Por tanto se puede recomendar el empleo de la cinta diametrica para el cálculo del área de la sección transversal, pues es más fácil de utilizarla y más barata.

\section{References:}

1. Clark, N. (2000). A Review of Past Research on Dendrometers.

2. Husch, B. (1982). Forest Mensuriation. New York: Jhon Wiley \& Sons.

3. Monroy, H. (2000). Manual de Planificación y Ejecución de Aprovechamienos Forestales en las Concesiones Comunitarias de Petén. Guatemala: CATIE.

4. Ramírez, C., \& Kleinn, C. (Marzo de 2001). fao.org. Obtenido de www.fao.org/forestry/download/15545-

0388865e6fb22f1d7d010789690304ac3.pdf

5. Ramirez, F. (1995). CDA. Obtenido de CDA: http://iniridaguainia.gov.co/apc-aa-

files/30616138616630333033656364306431/presentacioncubicacion-de-maderas.pdf

6. Robertson, F. (2000). Timber Cruising Handbook. USDA Forest Service.

7. Ugalde, L. (1981). Conceptos Básicos de Dasometria. En L. Ugalde, Conceptos Básicos de Dasometria (pág. 2). Turrialba: Bib. Orton IICA / CATIE.

8. Van Laar, A. a. (1997). Forest Mensuration. Cuvillier Verlag, 1-418.

9. Salas E, Christian; Reyes, Mauricio \& Bassaber E, Claudia (2005). Medición de diámetros fustales con relascopio y forcípula finlandesa: efectos en la estimación de volumen. Bosque (Valdivia) [online]. 2005, vol.26, n.2 [citado 2016-10-11], pp.81-90. Disponible en: <http://www.scielo.cl/scielo.php?script=sci_arttext\&pid=S071792002005000200010\&lng=es\&nrm=iso >.

ISSN $0717-$ 9200. http://dx.doi.org/10.4067/S0717-92002005000200010.

10. PRODAN, M. (1997). Mensura Forestal. Costa Rica. GTZ, IICA. 